\title{
Congenital Moulding Depressions of the Skull
}

\author{
J. H. M. AXTON,* M.B., B.S. ; L. F. LEVY, $\dagger$ M.SC., F.R.C.S., F.A.C.S.
}

Brit. med. F., 1965, 1, 1644-1647

Depressed fractures, or congenital moulding depressions, of the skull in the newborn are uncommon. It is accepted that these depressions are of two types: the majority are caused by forceps or digital pressure from the obstetrician's hand, while a small number are due to extreme moulding of the foetal skull to permit its passage through the mother's pelvis. The latter appear to be extremely rare, since a search of the literature reveals only 18 casés-five in America (Ingraham and Matson, 1954 ; Rawl, 1957 ; Pike, 1958) and 13 in French journals (Paillas et al., 1955;. Thurel and Baldacci, 1957)-although passing reference is made to them in textbooks of paediatrics and neurosurgery.

In the past three years 31 cases have been seen in the Neurosurgical Unit of the Harare Central Hospital, Salisbury, Rhodesia. All these cases occurred in African women, delivered at the Harare Hospital Maternity Centre, at hospitals served by Harare Hospital, or unattended at their homes. In only three cases was there any obstetrical intervention, and in each one it seems doubtful that the intervention caused the depression. We estimated an approximate and somewhat high incidence of one in 4,000 births, while during the same period (three years) one instrumentally produced depression was observed in deliveries of 6,000 European women at the Lady Chancellor Maternity Hospital. In this country, therefore, spontaneous moulding depressions appear to be relatively common among African women. It is because of this, and its rarity in Europeans, and also the paucity of discussions in the literature, that our attention has been focused on this condition, both as a medical curiosity and from the aspects of prevention and cure.

The condition commonly consists of a depression varying in size from a small groove to an indentation of the entire half of the frontal bone, running from the supraorbital ridge up to but never passing beyond the coronal suture (Figs. 1 and 2), although other bones may be involved. Tracz (1960) points out that in most cases these are not true fractures but indentations of malleable bone without loss of continuity, and he postulates that they may be caused by pressure of the foetal skull from any of the following: the symphysis pubis, the sacral promontory, a deformed pelvis, obstetrical forceps, or the obstetrician's hand during manual rotation.

Method.-In as many cases as possible the following observations were made: maternal $x$-ray pelvimetry, foetal skull diameters and circumferences, length of labour, birth weight, and foetal presentation. Previous obstetrical histories were rather difficult to obtain because many mothers had their deliveries unattended at home.

\section{Resuits}

In 17 cases out of 31 the depression was found in the frontal region on the same side of the head as that to which the occiput pointed during the first stage of labour (see Table). In one further case the depression was found in the parietal region of the corresponding side, and in all these cases it is believed that the depression was caused by the sacral promontory as the head descended past it to enter the pelvis. For example, in the case \footnotetext{
* Formerly house-surgeon, Harare Central Hospital, Salisbury, Rhodesia.
Present address : Hillingdon Hospital, Middlesex.

t Consultant Neurological Surgeon, Harare Central Hospital, Salisbury, Rhodesia.
}

of a left occipito-anterior presentation, the left fronto-parietal region of the foetal head will be in direct contact with the sacral promontory during the first stage of labour.

In three cases the side of the depression in the frontal region was opposite to that of the position of the child, and here the mechanism is obscure. In four cases the presentation was occipito-posterior, and in one of these the depression was felt ${ }^{\circ}$

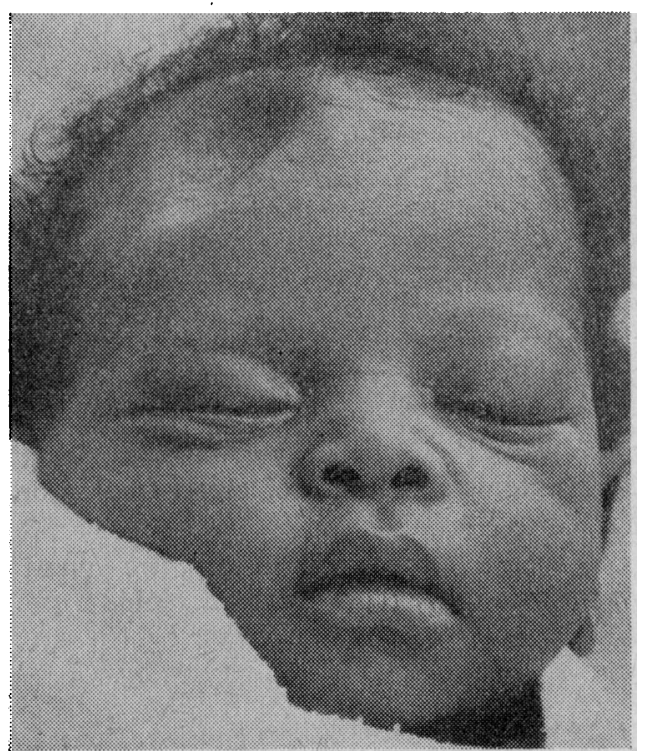

FIG. 1.-Case 3. One-week-old child, showing depression in a typical position in right frontal region. Vertex presentation, left occipito-anterior.

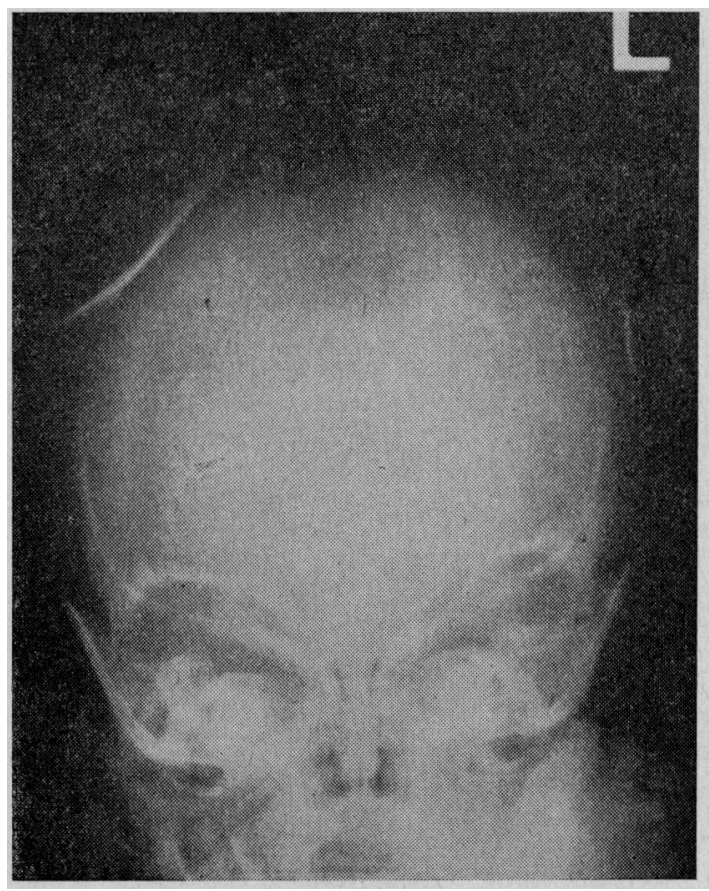

FIG. 2.-Case 3. Radiograph showing appearance of typical depression in right frontal region. 
against the pubic rami prior to an easy manual rotation and forceps extraction, so presumably it was an increased prominence of the pubic rami that was responsible. In another a child lying in the R.O.P. position in a mother with a gynaecoandroid pelvis turned itself spontaneously to R.O.A. A left frontal depression was noted, again presumably from pressure of the pubic ramus. A third mother whose child was lying in the R.O.P. position had a baby with a right frontal depression, and here, and in the fourth case, about which there is insufficient information, the mechanism is obscure. Both mothers had anthropoid pelves, and we think it likely that the pubic rami were the cause. In the remaining six cases the site of the depression was known but the position was not. Five of these depressions were frontal and one was parietal. There were no breech presentations.

Forceps were applied in three cases and theoretically could have caused the deformity, but we think this unlikely. In Case 21 forceps were applied easily for a left lateral lie in a small but normally shaped pelvis, but the child could not be delivered and caesarean section was performed. The depression, however, was in the characteristic high frontal position on the right side of the baby's head, and we assume it was caused by a pubic ramus, or sacral promontory if the presentation was wrongly interpreted. In Case 22 the depression was felt against the left pubic ramus by the examining obstetrician prior to the application of forceps. In Case 7 forceps were applied normally for delay in the second stage in a small and anthropoid pelvis. Since the depression in the right frontal region corresponds with the position, the sacral promontory seems the most likely cause. Three infants were delivered by caesarean section (Cases 5, 11, and 21).

The average birth weights of 24 of these infants were compared with the birth weights of 1,089 consecutive deliveries at the Harare Hospital, and statistically there was found to be no significant difference between the two groups. However, the head circumference and suboccipito-frontal and interparietal diameters were all found to be significantly larger when compared with a control group of 25 full-term normal deliveries. Of all these measurements the most significantly larger was the interparietal diameter. It should be stressed, however, that most of these measurements were made several days after birth, whereas those of the control group were made within 24 hours of birth.

It seems likely that increase in head size aggravates a relative cephalo-pelvic disproportion, but it is not the primary factor in producing the depression, as all the pelves measured showed some abnormality. Case 31 illustrates this well. A para-7 mother was delivered of the first of twins at her home. This child weighed $3 \mathrm{lb} .12 \mathrm{oz}$. (1,700 g.), had a head circumference of $12 \mathrm{in.}\left(305 \mathrm{~cm}\right.$.), interparietal diameter of $3 \frac{1}{4} \mathrm{in} .(8.3 \mathrm{~cm}$.), and a suboccipito-frontal diameter of 4 in. $(10 \mathrm{~cm}$.), and delivery was easy. The mother then came to hospital because there was a considerable delay in the delivery of the second child. Labour started spontaneously after admission, and she was delivered without assistance. The second baby weighed $5 \mathrm{lb} .6 \mathrm{oz}$. $(2,438$ g.) and had a head circumference of $13 \frac{3}{4}$ in. $(35 \mathrm{~cm}$.), I.P.

Details of Cases

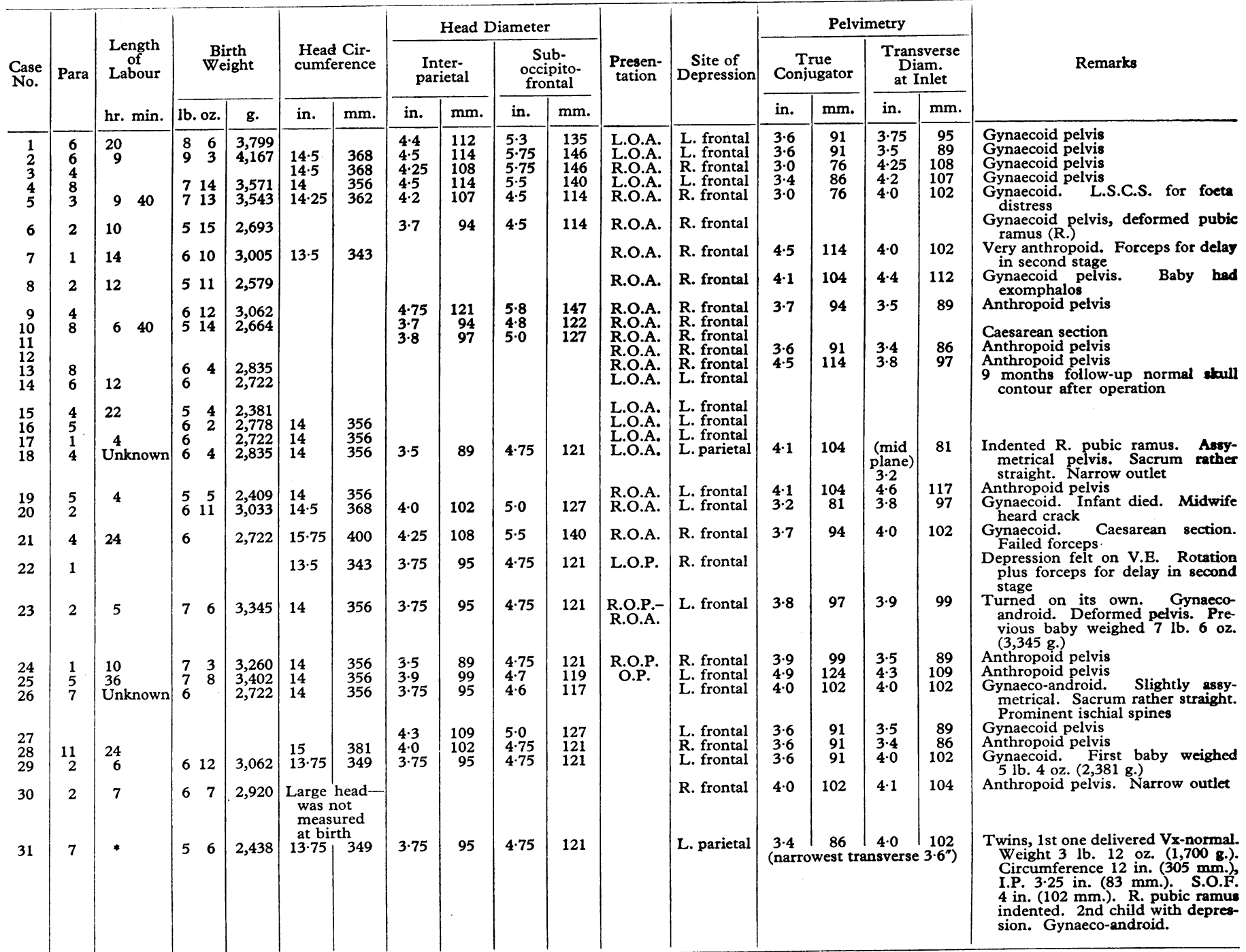


diameter of $3 \frac{3}{4}$ in. $\left(9.5 \mathrm{~cm}\right.$.), and S.O.F. diameter of $4 \frac{3}{4}$ in. $(12 \mathrm{~cm}$.). This child had a depression in the left parietal region, but unfortunately the position was not noted. The mother had a gynaeco-android pelvis with an inward deformity of the right pubic ramus.

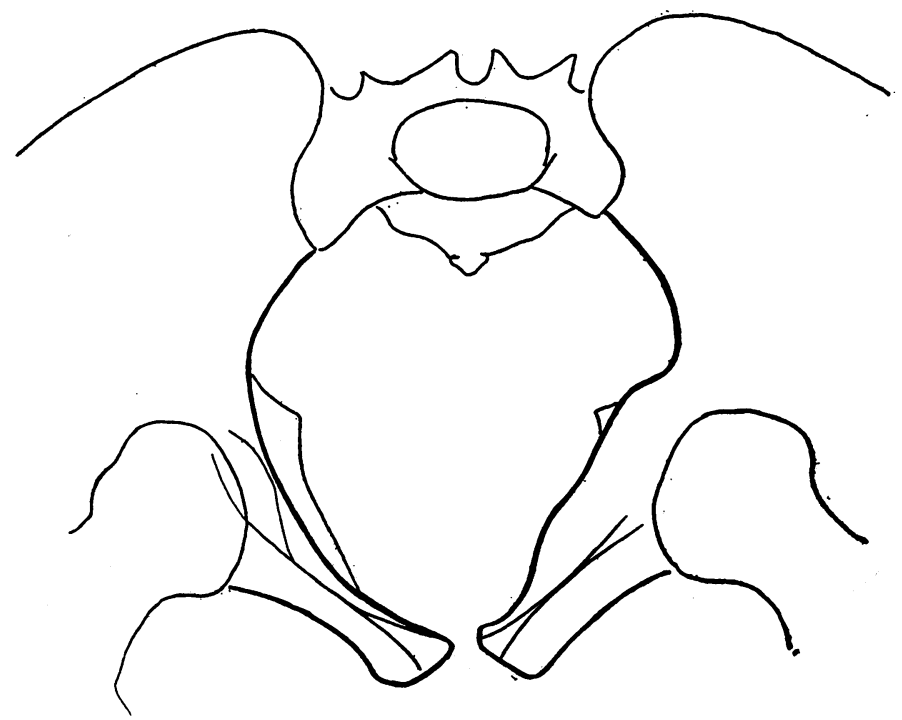

Fig. 3.-Asymmetrical pelvis of mother of Case 18. This mother had four children, the last three of whom were born with depressions in the temporo-parietal region (Fig. 4).

$X$-ray pelvimetry was undertaken in 23 cases and a radiograph of the pelvis in one further case. Eleven pelves were gynaecoid, nine markedly anthropoid, three gynaeco-android, and one was asymmetrical (Fig. 3). By accepted standards all these pelves would be small, but not enough pelvimetries have been performed locally to give a normal range. In three cases there was a marked deformity of one pubic ramus (Cases 6, 23, and 31). In Case 6 this was due to an old injury and in the other two rickets was a possible cause.

The average length of labour in our series did not differ statistically from the length of labour of 100 consecutive fullterm normal deliveries in the Harare Maternity Hospital.

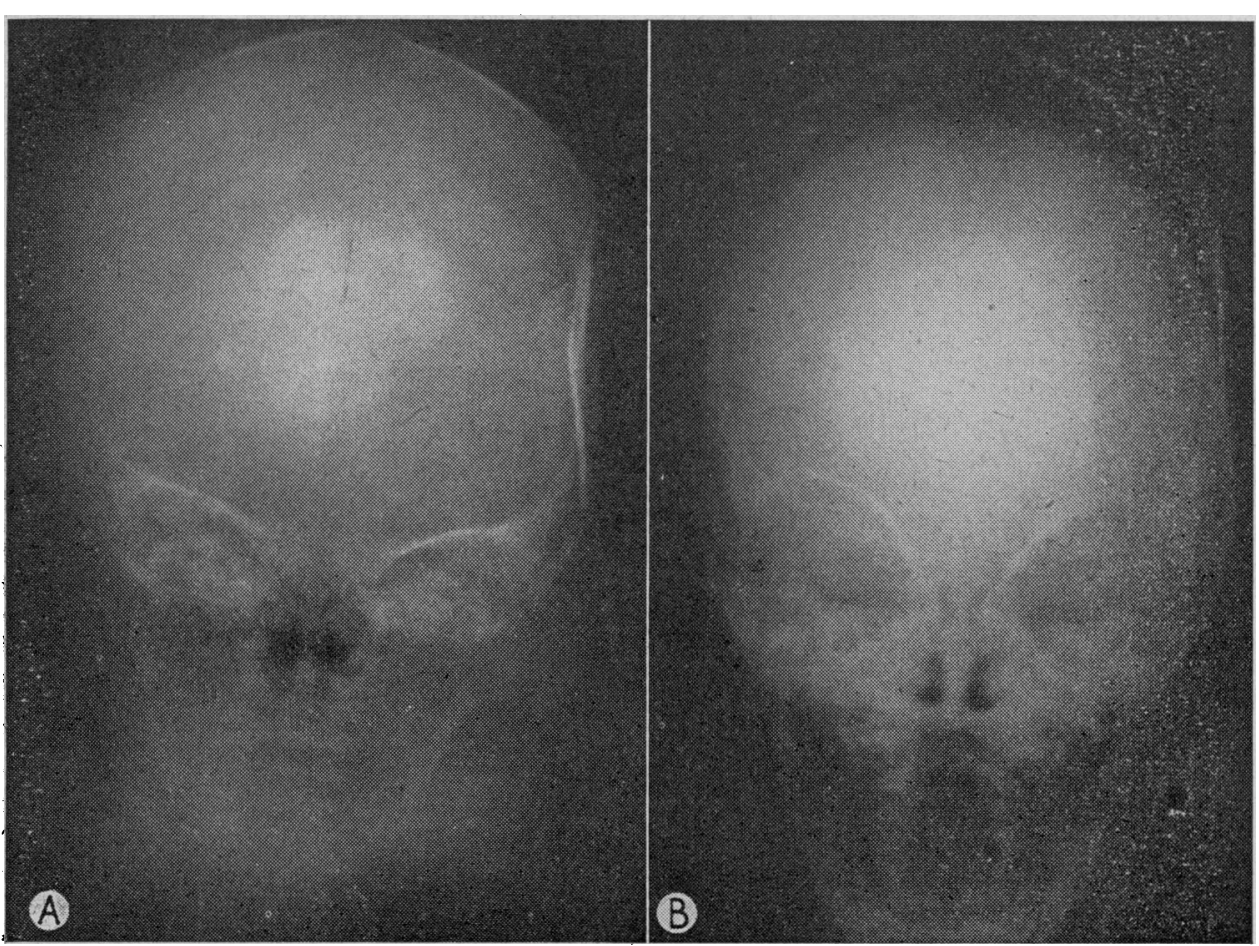

Fig. 4.-Case 18. Radiographs of skull of fourth child taken shortly after birth (A), and seven weeks later (B), showing considerable spontaneous elevation of the depression, which is virtually not noticeable to clinical examination.
In the 28 cases of known parity only four depressions occurred in first-born infants. This is probably due to an increase in the size of the foetus with successive pregnancies, making relative cephalo-pelvic disproportion worse-thus the first child may escape a depression by being small enough to go easily through the pelvis. In Case 29 the first child weighed $5 \mathrm{lb}, 4 \mathrm{oz}$. (2,381 g.) and was normal, the second child born one and half years later weighed $6 \mathrm{lb} .12 \mathrm{oz}$. $(3,060 \mathrm{~g}$.$) and had$ a depression. However, weight alone is not the only factor, since in Case 23 a previous baby of the same weight as the affected one was born without a depression. A second factor may be the tendency for multiparous women to push harder, which gives less time for the head to mould. This explains how these women manage to deliver their babies normally, with depressions, rather than to pass into obstructive labour and require caesarean section. In Case 20, where the cervix was fully dilated with the foetal head not yet engaged, the midwife heard a loud crack, coincidental with a very strong uterine contraction, and a few moments later the infant was born with a depression in the left frontal region. The child subsequently showed signs of cerebral irritation and died three weeks later despite elevation of the depression-the only death in this series.

In three cases two or more affected children have been born to one mother: in Case 28 the last two babies of a para-11 mother, in Case 6 both children of a para- 2 mother, and in Case 18 the last three of a para-4 mother. All these latter children had depressions in the temporo-parietal region rather than in the frontal region. One mother, not in this series, gave birth to all four of her children with a right frontal depression. She is well known to the midwives, but unfortunately could not be traced for pelvimetry. It appears that once a woman has had an affected baby the chances of her later children being affected are increased. However, one mother, after 10 normal deliveries, gave birth to a baby with a depression, yet the twelfth child was unaffected (Case 28). The relative weights of these children is not known, but the mother's pelvis was markedly anthropoid, and the depression in the sole affected baby could be accounted for only by a failure of asynclitism.

A further cause for the increased incidence in multiparous women could be a lack of calcium in the diet. The foetal skulls would be less hard than normal, and this would get worse with each succeeding pregnancy, but we have been unable to confirm this.

\section{Treatment}

We advised elevation of these depressions in order to minimize the possibility of brain-scarring from pressure, to exclude coexistent subdural effusions, and for cosmetic reasons. However, we did not consider this essential, as the majority corrected themselves over the course of a few months, and since this prognosis was well known to the parents it was not always possible to obtain permission for operation.

In the eight babies in whom elevation was carried out, the procedure was to drill a burr-hole or open up a suture line next to the depressed area, and, after working an elevator between the bone and the dura, to exert an outward force at the point of maximum depression. If this method failed, as it usually did, the bone was removed piecemeal and the fragments were replaced. Satisfactory bone formation was found in 
every case. Dural tears were observed in two cases but were not severe ; nonetheless, the existence of these tears in the occasional case makes elevation by placing an instrument between the bone and dura an unsatisfactory procedure, as further damage to the underlying brain could occur. There has been one death from diffuse brain damage (Case 20) associated with very rapid delivery. However, all the other children were neurologically normal on examination both before and after operation, and no abnormality was found in any of the unoperated cases, either at birth or when followed up. No subdural haematoma was found in any of the operated cases, and this suggests that the depression takes the place of excessive moulding with its attendant danger to the venous sinuses.

Three unoperated cases from this series and the two siblings of Case 18 showed no external signs of depression when followed up one or two years later, while in three cases (Nos. 18,25 , and 30 ) the depressions were obviously getting smaller (Fig. 4). Eighteen other operated cases have not been followed up. The four siblings mentioned previously have not been seen, but are reputed by the midwives to have undergone spontaneous correction within a year of birth.

\section{Discussion}

These cases support Rickham's (1961) contention that the depressions do not need elevation unless they are associated with symptoms of underlying brain damage or unless the parents wish it for cosmetic reasons. Holt and McIntosh (1953) have seen a number of these cases treated conservatively with no sequelae, while De Lee (1955) agrees that the depressions disappear almost entirely in a few months. Parsons and Barling (1954) recommend early operation in severe cases, although they state that shallow ones disappear spontaneously. Ingraham and Matson (1954) also recommend early operation. Thurel and Baldacci (1957) state that a late complication of unoperated depressions is an oval-shaped opening in the cranial vault found in association with epilepsy. We have been unable to confirm this owing to the difficulty of tracing patients, but have seen one patient with hydrocephalus who had an oval-shaped opening in the right frontal region. The mother stated that the child had suffered an injury to that part at or shortly after birth.

The diagnosis was made after birth in all our cases except one (Case 22) where the depression was felt by the obstetrician on vaginal examination. Rawl (1957) recalls two further cases where the diagnosis was made before birth, in one of which an $x$-ray film was taken when the mother went into obstructed labour. A depression could be seen against the sacral promontory and fifth lumbar vertebra. Pike (1958) records a similar case in the baby of a small woman (108 lb.; $49 \mathrm{~kg}$.) who fell down the stairs, hitting her lower abdomen against a step, when she was eight and a half months pregnant. After a short labour the baby was found to have a right frontal depression in the characteristic position. Pike postulates that the depression was caused by the blow forcing the foetal head against the maternal sacrum, but goes on to wonder why there was no serious damage to the maternal structures between the occiput and the edge of the step. It is possible that in such a small woman the depression could have been produced during labour, the blow being incidental. As in Pike's case, none of the mothers in our series suffered any damage. This is surprising, as vesicovaginal fistula is a common condition in African women, presumably resulting from low tissue resistance, due to poor nutrition.

Prevention of this condition would seem to be very diffcult in Rhodesia at present. Many women arrive at hospital in labour, unbooked, and even if cephalo-pelvic disproportion is suspected clinically there would be no time to confirm this radiologically. Only in women who have had previously affected children is one able to forecast the birth of a similarly affected child. It seems doubtful if caesarean section is warranted in these cases, as the resulting deformity to the child is so slight and transient.

\section{Summary}

Thirty-one cases of congenital moulding depressions of the skull are presented. The mechanism of production of these depressions was by pressure from the maternal sacral promontory in at least 18 cases, and pressure from the pubic ramus in a further four cases. Forceps were not responsible for any cases.

Of the 24 pelvimetries performed all were found to be abnormal in some way. Eleven were gynaecoid but small, nine were anthropoid, three gynaeco-android, and one was asymmetrical. Three of these were further abnormal in having deformed pubic rami.

Contributing factors, such as increased foetal head size and the possibility of lack of calcium in the diet giving rise to soft foetal skulls, are discussed. The absence of abnormal neurological signs, both immediately after birth and when followed up, is stressed.

We advised early operation, but did not insist on this, as spontaneous elevation usually occurred within a year.

We wish to express our thanks to the Secretary for Health, Rhodesia, for permission to publish this paper.

\section{REFERENCES}

De Lee, J. B. (1955). In Obstetrics, 11th ed., by J. P. Greenhill. Saunders, Philadelphia.

Holt, L. E., and McIntosh, R. (1953). Paediatrics, 12th ed. Appleton, New York.

Ingraham, F. D., and Matson, D. D. (1954). Neurosurgery of Infancy and Childhood. Thomas, Springfield, Ill.

Paillas, J. E., Bérard-Badier, M., Vigouroux, R., and Piganiol, G. (1955). Marseille chir., 7, 382.

Parsons, L. G., and Barling, S. G. (1954). Diseases of Infancy and Childhood, 2nd ed. Oxford Univ. Press, London.

Pike, J. B. (1958). Med. Tms (N.Y.), 86, 869.

Rawl, A. E. (1957). 7. S.C. med. Ass., 53, 44.

Rickham, P. P. (1961). Helv. chir. Acta, 28, 560.

Thurel, R., and Baldacci, Y. M. (1957). Sem. Hôp. Paris, 33, 3538.

Tracz, J. (1960). Clin. Proc. Child. Hosp. (Wash.), 16, 45. 